\title{
Patch-wise denoising of phase fringe patterns based on matrix enhancement
}

\author{
Rishikesh Kulkarni and Pramod Rastogi \\ Applied Computing and Mechanics Laboratory, Ecole Polytechnique Fédérale de \\ Lausanne, 1015 Lausanne, Switzerland
}

\begin{abstract}
We propose a new approach for the denoising of a phase fringe pattern recorded in an optical interferometric setup. The phase fringe pattern which is generally corrupted by high amount of speckle noise is first converted into an exponential phase field. This phase field is divided into number of overlapping patches. Owing to the small size of each patch, the presence of a simple structure of the interference phase is assumed in it. Accordingly, the singular value decomposition (SVD) of the patch allows to separate the signal and noise components effectively. The patch is reconstructed only with the signal component. In order to further improve the robustness of the proposed method, an enhanced data matrix is generated using the patch and the SVD of this enhanced matrix is computed. The matrix enhancement results in an increased dimension of the noise subspace which thus accommodates more amount of noise component. Reassignment of the filtered pixels of the preceding patch in the current patch improves the noise filtering accuracy. The fringe denoising capability in function of the noise level and the patch size is studied. Simulation and experimental results are provided to demonstrate the practical applicability of the proposed method.
\end{abstract}

Keywords: phase fringe pattern; exponential phase field; enhanced matrix; singular value decomposition.

\section{Introduction}

Electronic speckle pattern interferometry (ESPI) is used extensively as an optical, non-contact type measurement technique in many applications such as in deformation analysis of object surface under load, measurement 
of thermal gradient, surface contour, etc. It is assisted by the phase shifting technique to obtain the wrapped phase map corresponding to the physical parameter under observation. In the case of digital holographic interferometry (DHI), a wrapped phase map is obtained from the numerically reconstructed holograms without any need of the phase shifting procedure. The phase map, generally termed as phase fringe pattern, contains $2 \pi$ phase discontinuities which need to be removed using the unwrapping operation in order to obtain a continuous phase map. However, these phase fringe patterns are often corrupted by the speckle noise. The presence of speckle noise makes the reliable phase unwrapping a difficult task. The main aim of any fringe filtering technique being to remove the speckle noise effectively while preserving the details of the fringe pattern pertaining to the true interference phase, a number of noise filtering techniques of the phase fringe pattern have been proposed, such as the anisotropic sine/cosine average filter [1], the local histogram-data-orientated filter [2], the tangent least-squares fitting filter [3] , the adaptive filter [4], etc. The fringe filtering techniques based on the use of regularized cost function with the complex-valued Markov random fields [5], windowed Fourier transform [6], local polynomial approximation of phase [7], localized Fourier transform [8] and 2D continuous wavelet tranform [9] have also been reported. The comparisons of few of the fringe filtering techniques can be found in [10, 11].

In this paper, we propose a new approach of fringe filtering by locally analyzing the fringe pattern. The proposed method is based on the filtering of the exponential phase field obtained using the wrapped phase map. In this approach, a small sized patch of the exponential phase field is considered at a time. Due to the small size of the patch, a valid assumption of a simple structure of the phase can be considered. This assumption allows us to effectively remove the noise in the given patch by decomposing it into the signal and noise subspace.

\section{Theory}

In any optical interferometric setup, a normalized exponential phase field corresponding to the phase fringe pattern is represented as

$$
\Pi(x, y)=\exp [j \varphi(x, y)] .
$$

In the above equation, $j=\sqrt{-1} ; x \in[0, K-1]$ and $y \in[0, L-1]$ represent the pixels along the rows and columns, respectively; $\varphi(x, y)$ is the interference 
phase.

In the proposed method, the exponential phase field is divided into a number of patches $\left(\boldsymbol{\Pi}_{p} \mathrm{~s}\right)$ each of size $N \times N$. Denoising of the exponential phase field is performed by analyzing the fringe pattern in a given patch at a time. The exponential phase field in the patch can be represented as

$$
\Pi_{p}=\left[\begin{array}{cccc}
\Pi(0,0) & \Pi(0,1) & \cdots & \Pi(0, N-1) \\
\Pi(1,0) & \Pi(1,1) & \cdots & \Pi(1, N-1) \\
\vdots & \vdots & \ddots & \vdots \\
\Pi(N-1,0) & \Pi(N-1,1) & \cdots & \Pi(N-1, N-1)
\end{array}\right]
$$

Note that the coordinates from $(0,0)$ to $(N-1, N-1)$ used in the above equation are local to the patch. An enhanced matrix $\boldsymbol{\Pi}_{e}$ is generated by appending the patch with its partitioned rows and columns as in [12]

$$
\boldsymbol{\Pi}_{e}=\left[\begin{array}{cccc}
\boldsymbol{\Pi}_{0} & \boldsymbol{\Pi}_{1} & \cdots & \boldsymbol{\Pi}_{N-P} \\
\boldsymbol{\Pi}_{1} & \boldsymbol{\Pi}_{2} & \cdots & \boldsymbol{\Pi}_{N-P+1} \\
\vdots & \boldsymbol{\Pi}_{q} & \ddots & \vdots \\
\boldsymbol{\Pi}_{P-1} & \boldsymbol{\Pi}_{P} & \cdots & \boldsymbol{\Pi}_{N-1}
\end{array}\right]
$$

where,

$$
\Pi_{q}=\left[\begin{array}{cccc}
\Pi(q, 0) & \Pi(q, 1) & \cdots & \Pi(q, N-Q) \\
\Pi(q, 1) & \Pi(q, 2) & \cdots & \Pi(p, N-Q+1) \\
\vdots & \vdots & \ddots & \vdots \\
\Pi(q, Q-1) & \Pi(q, Q) & \cdots & \Pi(q, N-1)
\end{array}\right]
$$

The variables $P$ and $Q$ are the parameters which decide the size of the enhanced matrix. The size of enhanced matrix is $P Q \times(N-P+1)(N-Q+1)$. A singular value decomposition of the enhanced matrix is performed as follows

$$
\Pi_{e}=\boldsymbol{U}_{e} \boldsymbol{\Lambda}_{e} \boldsymbol{V}_{e}^{*},
$$

where, ${ }^{*}$ represents complex conjugate operation; $\boldsymbol{U}_{e}$ and $\boldsymbol{V}_{e}$ are the $P Q \times$ $P Q$ and $(N-P+1)(N-Q+1) \times(N-P+1)(N-Q+1)$ sized matrices containing the left and right singular vectors, respectively; $\boldsymbol{\Lambda}_{e}$ is a rectangular matrix of size $P Q \times(N-P+1)(N-Q+1)$ containing non-negative singular values arranged in descending order along its diagonal.

Singular value decomposition has been used in many different applications for the purpose of data reduction. In SVD based data reduction approach, 
the singular values of the decomposed data matrix are arranged in the decreasing order. The components of the data corresponding to the higher singular values carry most of the features of the data matrix. Consequently, considering the components with the higher singular values and neglecting the lower singular values allows faithful reconstruction of the data. In the proposed method, the SVD of the enhanced matrix obtained from the patch of the exponential phase field is computed. In the case of small size of the patch, we can assume a simple linear or quadratic behavior for the fringes present in the patch. Accordingly, there exists a single highest singular value corresponding to this fringe pattern. The remaining singular values are much lower compared to the highest singular value. These lower singular values correspond to the noise present in the exponential phase field and which is, in general, spread uniformly throughout the space frequency plane. A noise free reconstruction of the exponential phase field in the given patch is achieved by retaining only the highest singular value and its associated singular vectors. The enhancement of data matrix increases the dimension of the noise subspace. Consequently, the noise component is effectively decomposed in the noise subspace and removed from the phase field. In order to faithfully denoise the exponential phase field at each pixel, the field is divided into a number of overlapping patches. This approach allows each pixel of the exponential phase field to be a part of more than one patch. The denoised exponential phase field at each pixel is thus a updated in each of its associated denoised patches.

The criteria for the selection of the values of $P$ and $Q$ has been mentioned in [12]. In the proposed method, we select the values of $P$ and $Q$ equal to $\min \left(10, \frac{N}{2}\right)$ in order to achieve the compromise between the sufficient noise subspace dimension and the computational cost. With the selected values of $N, P$ and $Q$, the denoised enhanced matrix is computed as

$$
\hat{\boldsymbol{\Pi}}_{e}=\boldsymbol{u}_{e}^{(1)} \boldsymbol{\Lambda}_{e}^{(1)}\left(\boldsymbol{v}_{e}^{(1)}\right)^{*}
$$

where, $\boldsymbol{\Lambda}_{e}^{(1)}$ is the highest singular value; $\boldsymbol{u}_{e}^{(1)}$ and $\boldsymbol{v}_{e}^{(1)}$ are the associated singular vectors. Finally, the denoised patch of the exponential phase field of actual size is computed using the equations (2) -(44).

For the further improvement in the performance of the proposed method, we propose to use a scheme wherein the noisy exponential phase field is updated each time a patch is denoised. In this scheme, the noisy patch of the phase field is replaced by its denoised version and it is used during the 
denoising of the subsequent patch. As the subsequent patch carries half of the pixels from the previously denoised patch, the effective noise present in the patch is less. This improves the performance of the proposed method.

The proposed method was implemented using MATLAB on a $2.93 \mathrm{GHz}$ Intel core i7 processor machine with 8 GB RAM. In [13], we had used the svd routine available in MATLAB to perform the SVD of the patch of the phase field. In the proposed method, we use the computationally efficient algorithm for SVD computation implemented in MATLAB [14].

\section{Numerical results}

The proposed method is numerically validated using a simulated phase fringe pattern of size $512 \times 512$ shown in Fig. 1(a). The scheme of the segmentation of the exponential phase field into overlapping patches is shown in Fig. 1(b). To evaluate the performance of the proposed method in function of the patch size and the noise level, simulations were performed in the presence of complex additive white Gaussian noise at different noise levels and with different patch sizes. We have used the root mean square error (RMSE) in the estimation of the unwrapped phase from the denoised phase fringe pattern as the evaluation criteria. The plot of the RMSEs in function of the signal to noise ratios (SNRs) expressed in $\mathrm{dB}$ and the patch sizes is shown in Fig. 1(c). The simulations were performed for each patch size, and the SNR and the RMSEs were computed. For illustration, the denoised phase fringe pattern computed at $\mathrm{SNR}=0 \mathrm{~dB}$ is shown in Fig. 1(d).

A simulation was performed in the presence of speckle noise in order to evaluate the performance of the proposed method in practical scenario. The speckle noise was simulated according to the method presented in [15]. A speckle noise corrupted phase fringe pattern with a speckle size greater than one pixel is shown in Fig. 2(a). The proposed method was implemented with the patch size of $16 \times 16$. The denoised phase fringe pattern shown in Fig. 2 (b) indicates the capability of the proposed method in denoising a highly noise corrupted fringe pattern.

The performance of the proposed method is compared with the windowed Fourier transform (WFT) technique proposed in [6]. The WFT based filtering has an effect of a low pass filtering wherein, a frequency band has to be specified as an input argument to the WFT algorithm. If a portion of the fringe pattern containing fringe frequencies higher than this band lies in the phase field, then this portion will not be faithfully denoised by the WFT 


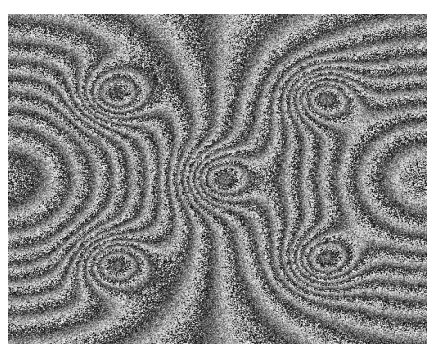

(a)

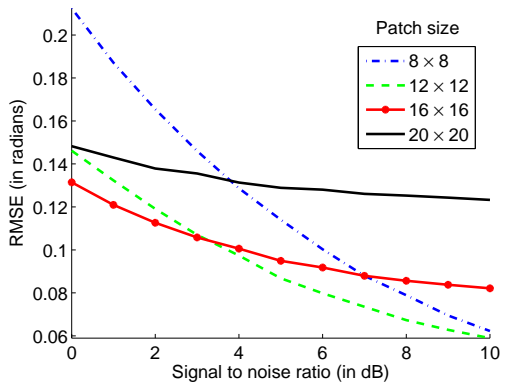

(c)

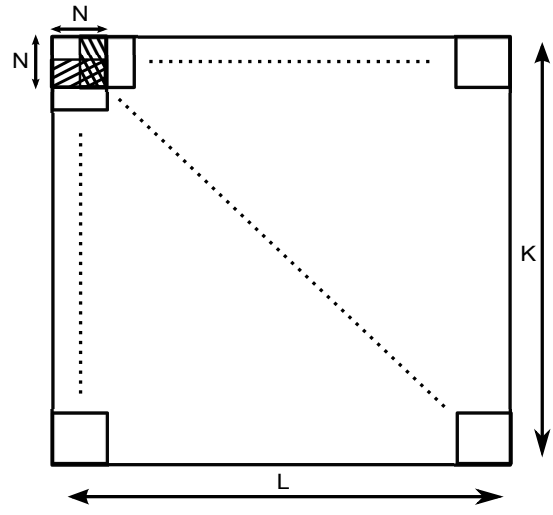

(b)

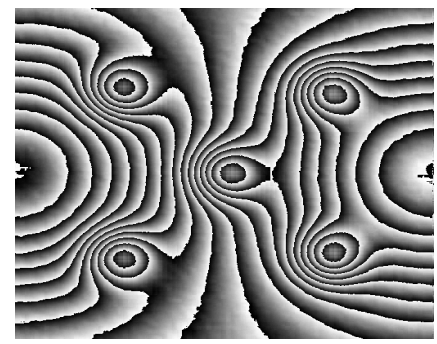

(d)

Figure 1: (a) Noisy phase fringe pattern at signal to noise ratio of $0 \mathrm{~dB}$. (b) Overlapped patching of exponential phase field used in the proposed method. (c) Root-mean-squareerrors in the estimated phase computed for different values of SNR and patch sizes (d) denoised phase fringe pattern.

algorithm. On the other hand, the proposed method in this paper is independent of the fringe frequency. This is one of the most important advantages of the proposed method. Consequently, the fringe pattern with widely varying fringe frequency can be denoised effectively using the proposed method with the given patch size. An example of such a fringe pattern is shown in Fig. 3(a). The denoised phase fringe pattern obtained using the proposed method with the patch size of $12 \times 12$ is shown in Fig. 3(b). For comparison, the WFT algorithm given in [6] was applied to the fringe pattern as $w f t(\Pi, 10,-0.5,0.1,0.5,10,-0.5,0.1,0.5,6)$. It can be clearly observed from Fig. 3(c) that whereas the region of high fringe density could not be denoised faithfully by the WFT algorithm with the selected frequency band, the pro- 


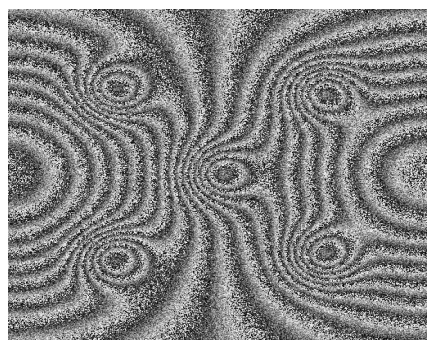

(a)

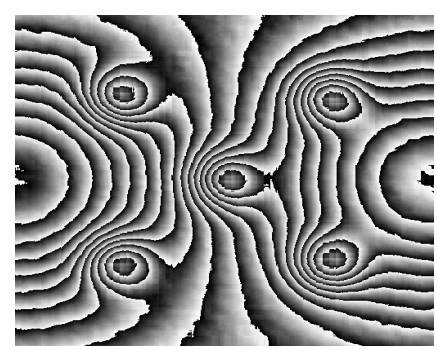

(b)

Figure 2: (a) Speckle noise corrupted phase fringe pattern and (b) denoised phase fringe pattern.

posed method successfully denoised the complete phase fringe pattern. For effective denoising of this high density fringe pattern using the WFT, a different frequency band for filtering has to be specified. This indicates that the selection of frequency band in using WFT depends on the fringe density, and which in turn varies the processing time. On the contrary, the selection of patch sizes and the corresponding processing time depends only on the noise level and not on the fringe density as such. The processing time of the proposed method with the patch size of $16 \times 16$ for denoising $512 \times 512$ sized phase fringe pattern was found to be 23.2 seconds.

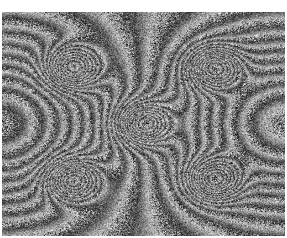

(a)

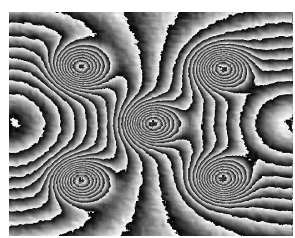

(b)

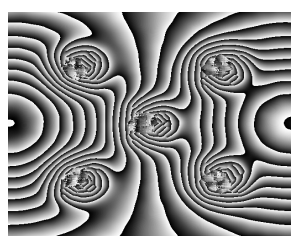

(c)

Figure 3: a) Noisy phase fringe pattern containing high density fringe areas (b) denoised phase fringe pattern using the proposed method with patch size $12 \times 12$ and (c) denoised phase fringe pattern using WFT.

\section{Experimental results}

A digital holographic interferometry set up was used to study the deformation of a square aluminum plate clamped along its edges. A laser light 
source of $532 \mathrm{~nm}$ wavelength was used to record the hologram of the object in its undeformed and deformed states. A SONY XCL-U1000 gray-scale camera was used to record the holograms. The exponential phase field is generated as the conjugate multiplication of the numerically reconstructed holograms. In the first experiment, deformation of a square plate containing a hole and clamped uniformly along its edges was studied. The plate was deformed using a point load applied at its center. The speckle noise corrupted phase fringe pattern of size $363 \times 315$ corresponding to the given deformation is recorded as shown in Fig. 4(a). The proposed method was implemented with the patch size of $12 \times 12$. The denoised phase fringe pattern is shown in Fig. 4(b).

In another experiment, a square plate without any hole and clamped nonuniformly along its edges was used. The phase fringe pattern of size $400 \times 400$ corresponding to the deformation caused by the point load applied to the plate's center is shown in Fig. 5)(a). The proposed method was implemented with the patch size of $16 \times 16$. The denoised phase fringe pattern shown in Fig. 5(b) indicates that the proposed method successfully removed the noise from highly noisy and dense fringe areas. The unwrapped phase estimate (in radians) is shown in Fig. 5(c) for the sake of illustration. In another experiment, a digital speckle pattern interferometry setup was used to study the in-plane deformation of a fiber reinforced wooden specimen. The recorded noisy phase fringe pattern of size $384 \times 336$ is shown in Fig. 5(d). The proposed method was implemented with the patch size of $16 \times 16$. The denoised phase fringe pattern is shown in Fig. 5(e). The unwrapped phase estimate (in radians) is shown in Fig. 5)(f).

\section{Conclusion}

A novel phase fringe pattern denoising technique is proposed. In this technique, the exponential phase field is divided into number of overlapping patches. The size of the patch depends on the noise level with larger patch size selected in the case of high noise level. The singular value decomposition based approach provides a robust and computationally efficient fringe denoising capability. The fringe filtering using the proposed method is independent of the fringe density which provides it a unique capability of denoising fringe patterns with highly varying fringe densities for the given patch size. Simulation and experimental results demonstrated the practical applicability of the proposed method. 


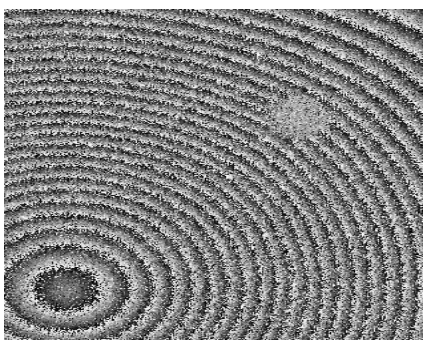

(a)

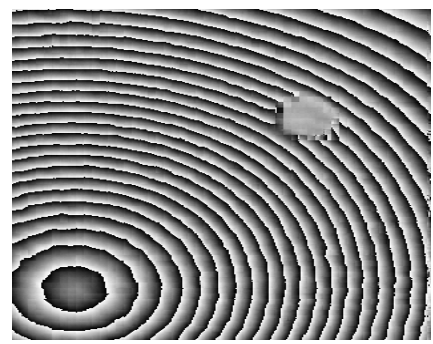

(b)

Figure 4: (a) Phase fringe pattern corresponding to the deformation of square plate recorded in a digital holographic interferometry setup and (b) denoised phase fringe pattern with the patch size $12 \times 12$.

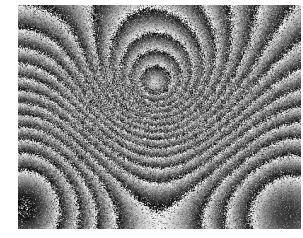

(a)

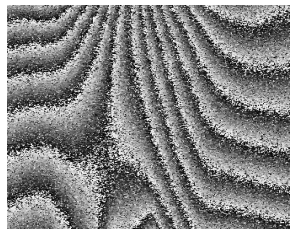

(d)

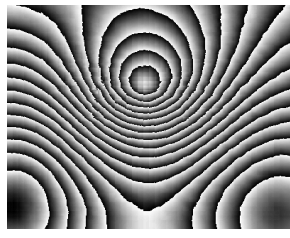

(b)

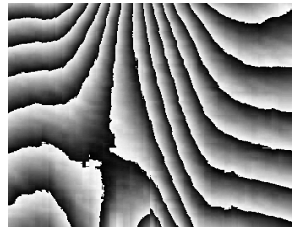

(e)

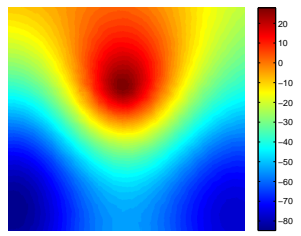

(c)

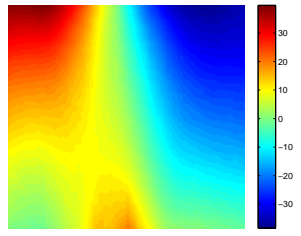

(f)

Figure 5: (a) Phase fringe pattern corresponding to the deformation of square plate recorded in a digital holographic interferometry setup (b) denoised phase fringe pattern with the patch size $16 \times 16$ (c) unwrapped phase estimate (in radians). (d) Phase fringe pattern corresponding to the in-plane deformation of a fiber reinforced wooden specimen recorded in a digital speckle pattern interferometry setup (e) denoised phase fringe pattern with the patch size $16 \times 16$ and (f) unwrapped phase estimate (in radians).

\section{Acknowledgments}

The authors would like to thank the Swiss National Science Foundation for its support provided under grant 200020_155937. 


\section{References}

\section{References}

[1] Aebischer H A, Waldner S. Simple and effective method for filtering speckle-interferometric phase fringe patterns. Opt Comm 1999;162(4):205-10.

[2] Huang M J, Sheu W. Histogram-data-orientated filter for inconsistency removal of interferometric phase maps. Opt Eng 2005;44(4):1-11.

[3] Tang C, Wang W, Yan H, Gu X. Tangent least-squares fitting filtering method for electrical speckle pattern interferometry phase fringe patterns. App Opt 2007;46(15):2907-13.

[4] Palacios F, Gonçalves E, Ricardo J, Valin J L. Adaptive filter to improve the performance of phase-unwrapping in digital holography. Opt Comm 2004;238(4-6):245-51.

[5] Villa J, Rodríguez-Vera R, Antonio Quiroga J, de la Rosa I, González E. Anisotropic phase-map denoising using a regularized cost-function with complex-valued markov-random-fields. Opt Lasers Eng 2010;48(6),65056 .

[6] Kemao Q. Two-dimensional windowed fourier transform for fringe pattern analysis: Principles, applications and implementations. Opt Lasers Eng 2007;45(2):304-17.

[7] Jose B, Katkovnik V, Astola J, Egiazarian K. Absolute phase estimation: adaptive local denoising and global unwrapping. App Opt 2008;47(29):5358-69.

[8] Li C, Tang C., Yan H, Wang L, Zhang H. Localized Fourier transform filter for noise removal in electronic speckle pattern interferometry wrapped phase patterns. App Opt 2011;50(24):4903-11.

[9] Escalante N., Villa J, De La Rosa I, De La Rosa E, González-Ramírez E, Gutiérrez O, Olvera C, Araiza M 2-D Continuous Wavelet Transform for ESPI phase-maps denoising. Opt Lasers Eng 2013; 51(9):1060-65.

[10] Kemao Q, Nam L T H, Feng L, Soon S H. Comparative analysis on some filters for wrapped phase maps. App Opt 2007;46(30);7412-18. 
[11] Tang C, Wang L, Yan H, Li C. Comparison on performance of some representative and recent filtering methods in electronic speckle pattern interferometry. Opt Lasers Eng 2012;50(8):1036-51.

[12] Hua Y. Estimating two-dimensional frequencies by matrix enhancement and matrix pencil. IEEE Tran Sig Proc 1992;40(9):2267-80.

[13] Kulkarni R, Rastogi P. Fringe filtering technique based on local signal reconstruction using noise subspace inflation. J Mod Opt 2016;63(6):18.

[14] Vijayan V. Fast SVD and PCA. http://www.mathworks.com/matlabcentral/fileexchange/47132-fast-svd-and-pca, MATLAB Central File Exchange. 2005; Retrieved October 27, 2015.

[15] Federico A, Kaufmann G H. Comparative study of wavelet thresholding methods for denoising electronic speckle pattern interferometry fringes. Opt Eng 2001;40(11):2598-2604. 